\title{
On the isomorphism between the medial axis and a dual of the Delaunay graph
}

\author{
Sharma, Ojaswa; Anton, François; Mioc, Darka
}

Published in:

Sixth International Symposium on Voronoi Diagrams, 2009. ISVD '09

Link to article, DOI:

10.1109/ISVD.2009.18

Publication date:

2009

Document Version

Publisher's PDF, also known as Version of record

Link back to DTU Orbit

Citation (APA):

Sharma, O., Anton, F., \& Mioc, D. (2009). On the isomorphism between the medial axis and a dual of the Delaunay graph. In Sixth International Symposium on Voronoi Diagrams, 2009. ISVD '09: ISVD 2009 (Vol. 6, pp. 89-95). IEEE. https://doi.org/10.1109/ISVD.2009.18

\section{General rights}

Copyright and moral rights for the publications made accessible in the public portal are retained by the authors and/or other copyright owners and it is a condition of accessing publications that users recognise and abide by the legal requirements associated with these rights.

- Users may download and print one copy of any publication from the public portal for the purpose of private study or research.

- You may not further distribute the material or use it for any profit-making activity or commercial gain

- You may freely distribute the URL identifying the publication in the public portal 


\section{On the isomorphism between the medial axis and a dual of the Delaunay graph}

\author{
Ojaswa Sharma, François Anton \\ Department of Informatics and Mathematical Modelling, \\ Technical University of Denmark, \\ Building 321, \\ 2800 Kgs. Lyngby, Denmark \\ $\{o s, f a\} @ i m m . d t u . d k$
}

\begin{abstract}
In this paper, we show a graph isomorphism between a dual graph of the Delaunay graph of the sampled points and the medial axis of the sampled features. This dual graph captures the fact that two Delaunay triangles share two vertices or an edge. Then, we apply it to the computation of the medial axis of the features selected in an image. The computation of the medial axis of images is of interest in applications such as mapping, climatology, change detection, medicine, etc. This research work provides a way to automate the computation of the medial axis transform of the features of color 2D images. In color images, various features can be distinguished based on their color. The features are thus extracted as object borders, which are sampled in order to compute the medial axis transform. We present also a prototype application for the completely automated or semiautomated processing of (satellite) imagery and scanned maps. Applications include coastline extraction, extraction of fields, clear cuts, clouds, as well as heating or pollution monitoring and dense forest mapping among others.
\end{abstract}

Keywords-Voronoi diagram; Delaunay graph; medial axis; graph isomorphism; topology;

\section{INTRODUCTION}

Popular methods for computation of the medial axis are thinning using mathematical morphology [1, chap. 9] and skeletonization using distance transform [2]. This research is concerned with computing the medial axis using a dual of the Delaunay graph, that captures the adjacency of internal Delaunay triangles along edges or vertices, together with the Voronoi diagram.

The work by Amenta et al. [3] leads to the extraction of object boundary from a set of sufficiently well sampled data points. The vertices of the Voronoi diagram approximate the medial axis of a set of sample points from a smooth curve. The vertices of the Voronoi diagram of the sample points were inserted into the original set of sample points and a new Delaunay triangulation was computed [3]. The circumcircles of this new triangulation approximate empty circles between the original boundary of the object and its skeleton. Thus, any Delaunay edge connecting a pair of the original sample points in the new triangulation is a part of the border [3]. The work by Amenta et al. [3] shows that the "crust" or the boundary of a polygon can be extracted from an unstructured set of points provided

\author{
Darka Mioc \\ Department of Geodesy and Geomatics Engineering, \\ University of New Brunswick, \\ Fredericton, N.-B., E3B 5A3, Canada \\ dmioc@unb.ca
}

the data points are well sampled. However it requires the additional computation of the Voronoi diagram after having added the Voronoi vertices of the original sample points as generators. It is thus a two step process.

Further research by Gold [4] leads to a one-step border (crust) extraction algorithm. In a Delaunay triangulation, each Delaunay edge is shared by two triangles and the circumcenters of these triangles are the Voronoi vertices. A Voronoi edge connecting these two circumcenters is the dual edge to the Delaunay edge considered here. According to [4], a Delaunay edge is a part of the border if it has a circle that does not contain any Voronoi vertex. Furthermore, those Delaunay edges that are not the part of the border set have their dual Voronoi edges as being part of the skeleton.

Gold and Snoeyink [5] further simplify their method and show that the boundary can be extracted in a single step. Gold [4] discusses about "anti-crust" in the context of skeleton extraction citing a brief introduction of this term in [3]. The idea behind getting the skeleton is that a Voronoi edge is a part of the skeleton if its corresponding dual Delaunay edge is not a part of the border set (crust) and it lies completely within the selected object. Thus, selecting the Voronoi edges lying inside the selected object that are dual of the non-crust Delaunay edges should give us the skeleton (see Figure 1). The Voronoi edges thus selected form a tree structure called the "anti-crust" [4], that extends toward the boundary but does not cross it.

The anti-crust of an object, as described above, forms a tree like structure that contains the skeleton. Once all the Delaunay edges belonging to the border set or the crust are identified using the condition given by [4], it is easy to identify the Voronoi edges belonging to the anti-crust. Figure 2 shows the Delaunay triangulation (dashed edges), the corresponding Voronoi diagram (dotted edges) and the crust edges (solid thick edges).

Once the anti-crust is identified, an appropriate pruning method can be applied to get rid of the unwanted edges, that are the main problem of this kind of approach. The "hairs" around the skeleton result from the presence of 


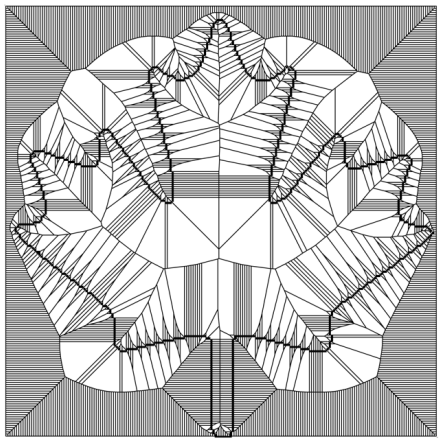

(a) The Voronoi diagram

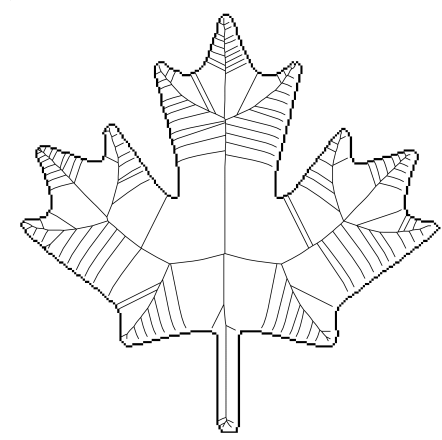

(b) Anti-crust

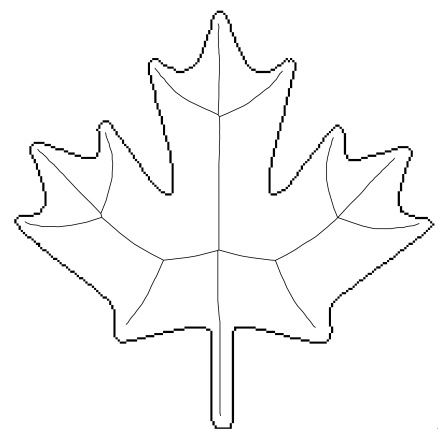

(c) Skeleton

Figure 1. Skeleton as seen as the anti-crust.

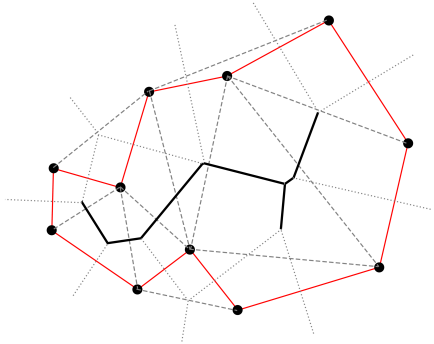

Figure 2. Anti-crust from the crust.

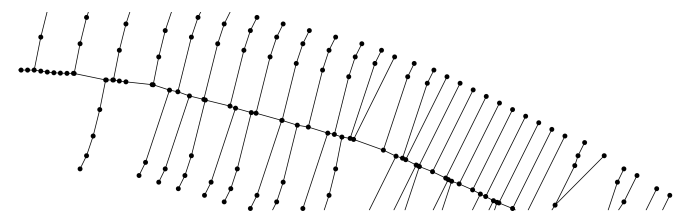

Figure 3. Hair around the skeleton composed of multiple edges.

three adjacent sample points whose circumcircle does not contain any other sample point - either near the end of a main skeleton branch; or at locations on the boundary where there is minor perturbation because of raster sampling [4] (see Figure 3). The problem of identifying skeleton edges now reduces to reasonably pruning the anti-crust. A skeleton retraction scheme suggested by [6] gets rid of the hairs and also results in smoothing of the boundary of the object. Ogniewicz [7] presents an elaborate skeleton pruning scheme based on various residual functions. Thus a hierarchic skeleton is created which is good for multiscale representation.

In this paper, we show the graph isomorphism between the medial axis of a 2D object and a newly defined dual graph of the Delaunay graph. The dual of the Delaunay graph considered here is the one with faces of the Delaunay triangles replaced by their isobarycenters. An edge in the dual graph joins two such isobarycenters of two adjacent triangles. Every vertex of the Delaunay triangulation has a corresponding polygon in the dual graph formed by the dual edges. We formulate rules to address singularities in computation of skeletons. The rules also handle degenerate cases where more than three vertices in the Delaunay graph are cocircular. Application of these rules gives rise to a valid and robust skeleton. We apply these rules to generate skeletons of various objects.

\section{PREliminaries}

Definition 1. (Medial axis) The medial axis of a closed and bounded set $X \subset \mathbb{R}^{2}$ is the set of all centers of maximal radius circles inscribed in $X$.

Let $\mathcal{P}=\left\{p_{i}, i=1, \ldots, m\right\}$ be a set of points of $\mathbb{R}^{2}$.

Definition 2. (Voronoi region) The Voronoi region $V\left(p_{i}, \mathcal{P}\right)$ of $p_{i} \in \mathcal{P}$ with respect to the set $\mathcal{P}$ is: $V\left(p_{i}, \mathcal{P}\right)=\{x \in$ $\left.\mathbb{R}^{2} \mid \forall p_{j} \in \mathcal{P}, p_{j} \neq p_{i} \rightarrow d\left(x, p_{i}\right)<d\left(x, p_{j}\right)\right\}$.

Definition 3. (Voronoi diagram) The Voronoi diagram of $\mathcal{P}$ is the union $V(\mathcal{P})=\bigcup_{p_{i} \in \mathcal{P}} \partial V\left(p_{i}, \mathcal{P}\right)$ of all Voronoi region boundaries.

Definition 4. (Delaunay graph) The Delaunay graph $D G(\mathcal{P})$ of $\mathcal{P}$ is the dual graph of $V(\mathcal{P})$ defined as follows:

- the set of vertices of $D G(\mathcal{P})$ is $\mathcal{P}$,

- for each edge of $V(\mathcal{P})$ that belongs to the common boundary of $V\left(p_{i}, \mathcal{P}\right)$ and of $V\left(p_{j}, \mathcal{P}\right)$ with $p_{i}, p_{j} \in \mathcal{P}$ 
and $p_{i} \neq p_{j}$, there is an edge of $D G(\mathcal{P})$ between $p_{i}$ and $p_{j}$ and reciprocally, and

- for each vertex of $V(\mathcal{P})$ that belongs to the common boundary of $V\left(p_{i_{1}}, \mathcal{P}\right), \ldots, V\left(p_{i_{4}}, \mathcal{P}\right)$, with $\forall k \in$ $\{1, \ldots\},, p_{i_{k}} \in \mathcal{P}$ all distinct, there exists a complete graph $K_{4}$ between the $p_{i_{k}}, k \in\{1, \ldots, 4\}$, and reciprocally.

\section{OUR MEDIAL AXIS APPROACH}

This is our main contribution where we exhibit the graph isomorphism between the dual graph of the Delaunay graph and the medial axis.

Since the vertices of the Delaunay graph are sample points located on the boundary of the object, the boundary of the objects is a subset of the Delaunay graph. Let us now consider the subgraph $I D G$ of the Delaunay graph $D G$ that lies in the interior or the boundary of the objects. Now consider the following dual graph $D I D G$ of the graph $I D G$, constructed by applying the following rules in order:

- the vertices of $D I D G$ are the isobarycenters of the vertices of each one of the triangles of $I D G$ that do not belong to a complete subgraph of at least 4 vertices of $I D G$ (that are cocircular) (see Fig 4(a));

- for each complete subgraph of at least 4 vertices of $I D G$ (that are cocircular), the corresponding subgraph of $D I D G$ is reduced to a point (see Fig 4(b));

- for each set $E$ of edges $e_{1} \ldots e_{k}, k>2$ of the boundary of $I D G$ that share one common vertex $v$ that is a vertex of a complete subgraph $K$ of at least 4 vertices of $I D G$, there is a set of edges of $D I D G$ that link $v$ to each one of the isobarycenters of the triangles $t_{1} \ldots t_{j}$ such that $t_{i} \in I D G$ and $t_{i}$ has two of its edges in $E$ that are not edges of $K$, and there is one edge of DIDG that links $v$ to the center of the circumcircle of the vertices of $K$ (see Fig 4(c));

- for each set $E$ of edges $e_{1} \ldots e_{k}, k>2$ of the boundary of $I D G$ that share one common vertex $v$ that is not a vertex of a complete subgraph of at least 4 vertices of $I D G$, there is a set of edges of $D I D G$ that link $v$ to each one of the isobarycenters of the triangles $t_{1} \ldots t_{j}$ such that $t_{i} \in I D G$ and $t_{i}$ has two of its edges in $E$ (see Fig 4(d));

- for each edge $e$ that is not on the boundary of $I D G$ and that does not link two vertices of a complete subgraph of at least 4 vertices of $I D G$, there exists an edge of $D I D G$ that links the isobarycenters of the vertices of each one of the triangles that share $e$ (see Fig 4(e));

Proposition 5. The graph DIDG is isomorphic to the medial axis of the boundary of IDG.

Proof: We call ramification vertices, the vertices of $D I D G$ that have a degree greater than 2 . We call dangling vertices, the vertices of $D I D G$ that have degree 1.

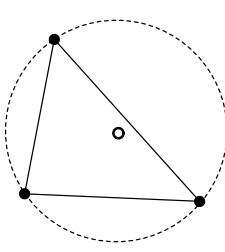

(a)

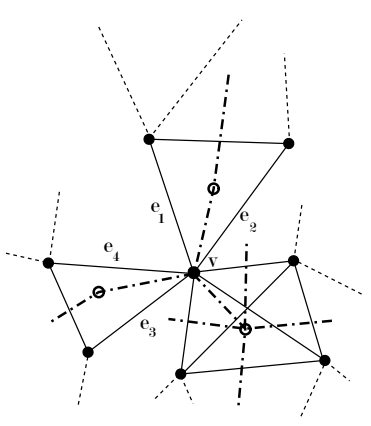

(c)

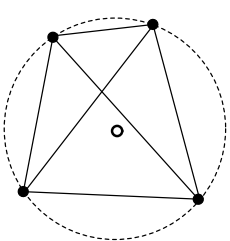

(b)

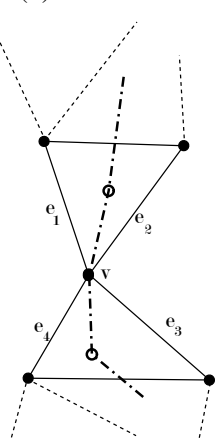

(d)

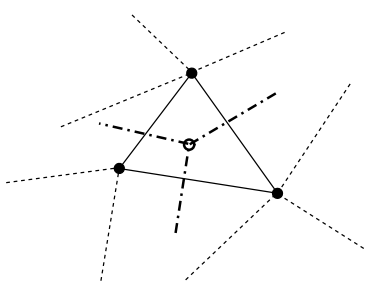

(e)

Figure 4. Rules.

- The dangling vertices of $D I D G$ correspond to triangles of $I D G$ that have two of their edges on the boundary of $D I D G$ (which are therefore adjacent). The corresponding vertices of the medial axis are the centers of maximal circles that touch two adjacent edges of the boundary of $I D G$ (see Fig 5(a)).

- The ramification vertices of $D I D G$ correspond either to the common vertex of a set $E$ of edges $e_{1} \ldots e_{k}, k>2$ of the boundary of $I D G$, or to the isobarycenters of the triangles of $D I D G$ that have no edge in the boundary of $I D G$. The later kind of ramification vertices (that we will call type I ramification vertices) correspond to Voronoi vertices that are at the same distance with respect to 3 distinct vertices on 3 distinct portions of the boundary of $I D G$. The earlier kind of ramification vertices (that we will call type II ramification vertices) correspond to singular points of the boundary of $I D G$ (see Fig 5(b)).

- The internal vertices on the paths between two type I ramification vertices of $D I D G$ correspond to triangles of $I D G$ that have one edge in the boundary of $I D G$. The edges in such paths link isobarycenters of triangles of $I D G$ that have their edge in the boundary of $I D G$ 
on different portions of the boundary of $I D G$. These edges correspond to edges of the medial axis, whose points are the centers of maximal circles that touch two different portions of the boundary of $I D G$ (see Fig 5(c)).

- The internal vertices on paths between one type I ramification vertices of $D I D G$ and a dangling vertex of $D I D G$ correspond to triangles of $I D G$ that have one edge in the boundary of $I D G$. Again, the edges in such paths link isobarycenters of triangles of $I D G$ that have their edge in the boundary of $I D G$ on different portions of the boundary of $I D G$. Again, these edges correspond to edges of the medial axis, whose points are the centers of maximal circles that touch two different portions of the boundary of $I D G$ (see Fig 5(d)).

- The internal vertices on paths between a type II ramification vertex and a type I ramification vertex correspond (like internal vertices between type I ramification vertices) to triangles of $I D G$ that have one edge in the boundary of $I D G$, except for the vertex that is connected to the type II ramification vertex by a single edge, which corresponds to a triangle of $I D G$ that has two of its edges on the boundary of DIDG (which are therefore adjacent). Again, the edges in such paths link isobarycenters of triangles of $I D G$ that have their edge in the boundary of $I D G$ on different portions of the boundary of $I D G$, or link the isobarycenter of a triangle that has two of its edges on the boundary of $I D G$. Again, these edges correspond to edges of the medial axis, whose points are the centers of maximal circles that touch either two different portions of the boundary of $I D G$, or two portions of the boundary of IDG that have a common singular vertex (see Fig 5(e)).

- The internal vertices on paths between a type II ramification vertex of $D I D G$ and a dangling vertex of $D I D G$ correspond (like internal vertices between type I ramification vertices) to triangles of $I D G$ that have one edge in the boundary of $I D G$, except for the vertex that is connected to the type II ramification vertex by a single edge, which corresponds either to a triangle of $I D G$ that has two of its edges on the boundary of $D I D G$ (which are therefore adjacent), or to the circumcircle of the vertices of a complete subgraph $K$ of 4 or more cocircular vertices of $I D G$. Again, the edges in such paths link isobarycenters of triangles of $I D G$ that have their edge in the boundary of $I D G$ on different portions of the boundary of $I D G$, or link the isobarycenter of a triangle that has two of its edges on the boundary of $I D G$, or link a singular vertex of $I D G$ with the center of a circumcircle of at leat 4 cocircular vertices of $I D G$. Again, these edges correspond to edges of the medial axis, whose points are the centers of maximal circles that touch either two different portions of the boundary of IDG, or two portions of the boundary of $I D G$ that have a common singular vertex (see Fig 5(f)).

Thus, the graph $D I D G$ is isomorphic to the medial axis of the boundary of $I D G$.

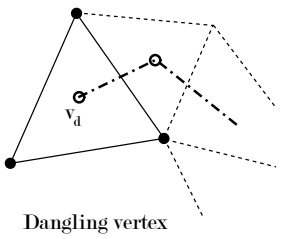

(a)

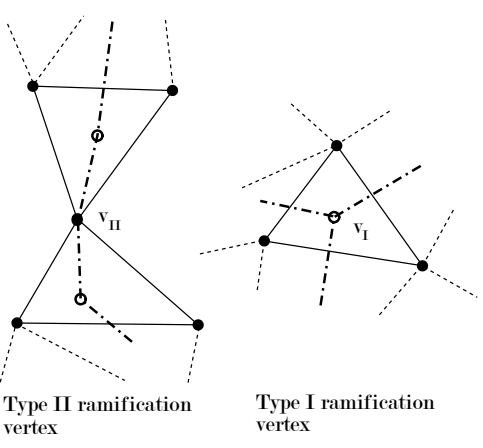

(b)

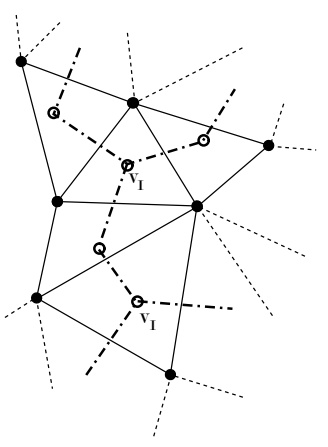

(c)

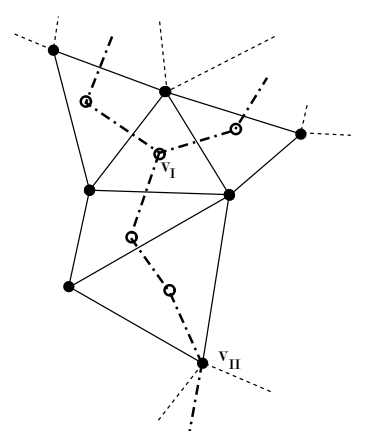

(e)

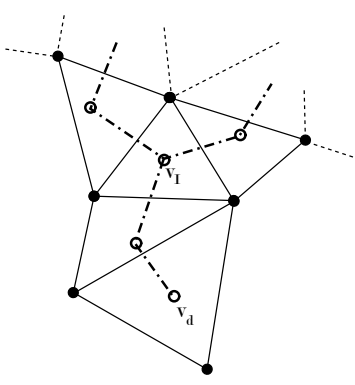

(d)

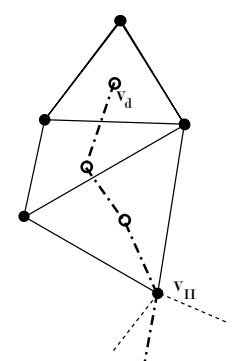

(f)
Figure 5. Proposition.

\section{Segmentation}

The segmentation method adopted here is the one provided by [8] which is based on feature space analysis.

Feature space analysis is used extensively in image understanding tasks. [8] provide a comparatively new and efficient segmentation algorithm that is based on feature space analysis and relies on the mean-shift algorithm to robustly determine the cluster means. A feature space is a space of feature vectors. These features can be object 
descriptors or patterns in the case of an image. As an example, if we consider a color image having three bands (red, green, and blue), then the image we see as intensity values plotted in Euclidean $\mathrm{XY}$ space is said to be in image space. Consider a three dimensional space with the axes being the three bands of the image. Each color vector corresponding to a pixel from the image can be represented as point in the feature space.

\section{Automated APPROACH TO SKELETONIZATION}

The general approach adopted here is:

1) Segment a color image into prominent objects.

2) Ask the user if he or she wants to process all the objects independently (automatic process) or select an object (semi-automatic process).

3) Collect sample points for each object to be processed.

4) Construct the Delaunay triangulation and its dual from the sample points.

5) Extract the medial axis using rules provided in section III.

Once objects are defined as homogeneous regions by the segmenter, the next step is to either select them all or some of them. To achieve this, the user is allowed to select a region on the image. If an object is composed of more than one regions then multiple object selection can be made and regions combined to form a single object. A wrongly selected region can be removed from the selection. The user input is processed and the selected region is highlighted and selected for next processing.

Once we have an object or all the objects chosen from an image, the next step is to sample its boundary in order to generate points used to construct the Delaunay triangulation. The Delaunay triangulation of the sample points is computed using the incremental algorithm given by [9] which is stored in the quad-edge data structure. This is followed by computation of the Voronoi vertices for all faces of the triangulation. The boundary of the object is extracted using the criteria given by [4]. The dual of the Delaunay graph mentioned in Section 2 is computed. The medial axis is obtained by replacing the edges of the dual of the Delaunay graph by corresponding edges of the Voronoi diagram.

\section{TIME COMPLEXITY}

Comaniciu $[10$, p. 21] shows that the complexity of the probabilistic mean shift type algorithm that is employed in the segmentation algorithm [8] is $O(m n)$, with $m \ll n$ where $n$ is the number of pixels in the input image (or the number of feature vectors in the feature space) and $m$ is the number of vectors in the initial feature palette or clusters. Comaniciu [10, p. 29] claims that the segmentation algorithm is linear with the number of pixels in the image. The complexity of the computation of the Delaunay graph $I D G$, the dual $D I D G$ and the medial axis is $O(l \log l)$ where $l$ is the number of sampled pixels. Overall, the time complexity can be said to be $O(\max \{m n, l \log l\})$.

\section{RESULTS}

We present the result of the computation of medial axis on different kinds of images: a scanned map (selected objects, see Fig 6) and a remote sensing image (all the objects, see Fig 7). The skeleton is shown in black in Fig 6(c) and Fig 7(c) along with the delaunay graph and the dual graph introduced in this paper. The dangling edges of the skeleton corresponding to the artifacts of sampling can be easily removed by removing the dangling branches of the dual graph of the Delaunay graph consisting of only one triangle barycenter and its edge to the main branch. Further, Fig 6 shows some missing information. The missing bits in the scanned map example could be obtained if the user selectes all the features in the application program.

\section{CONCLUSIONS}

We show in this paper how the medial axis of an object can be derived by following simple rules to avoid disconnected and degenerate skeletons. We showed that the resulting skeleton extracted using these rules are isomorphic to the medial axis. Further we design an effective methodology for automated vectorization and simplification of features in color images and implement our medial extraction in it. The methodology enables the automated extraction of the boundaries and the medial axis of an object in a single step. Coastline delineation, snow cover mapping, cloud detection, and dense forest mapping are a few areas where satisfactory results can be obtained.

\section{REFERENCES}

[1] R. C. Gonzalez and R. E. Woods, Digital Image Procesisng, 2nd ed. Prentice Hall, 2002.

[2] G. Borgefors, "Distance transformations in arbitrary dimensions," Computer Vision, Graphics, and Image Processing, vol. 27, no. 3, pp. 321-345, 1984.

[3] N. Amenta, M. Bern, and D. Eppstein, "The crust and the $\beta$-skeleton: Combinatorial curve reconstruction," Graphical models and image processing: GMIP, vol. 60, no. 2, pp. 125135, 1998.

[4] C. M. Gold, "Crust and anti-crust: A one-step boundary and skeleton extraction algorithm," in Symposium on Computational Geometry. New York, NY, USA: ACM Press, 1999, pp. 189-196.

[5] C. M. Gold and J. Snoeyink, "A one-step crust and skeleton extraction algorithm," Algorithmica, vol. 30, no. 2, pp. 144163, Jun 2001.

[6] C. M. Gold and D. Thibault, "Map generalization by skeleton retraction," in Proceedings of the 20th International Cartographic Conference (ICC), Beijing, China, August 2001, pp. 2072-2081. 


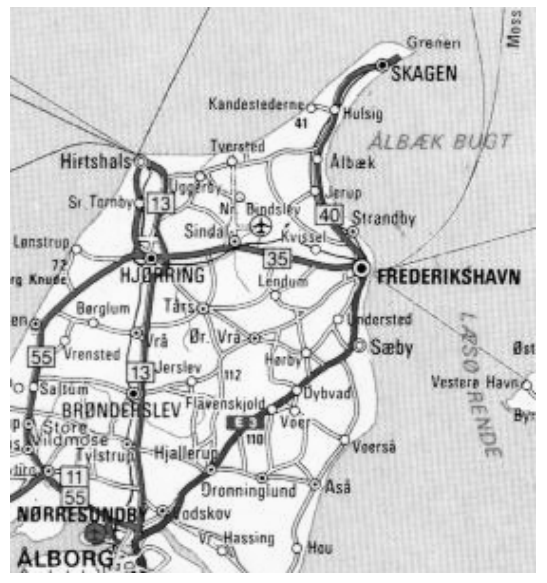

(a) Original image

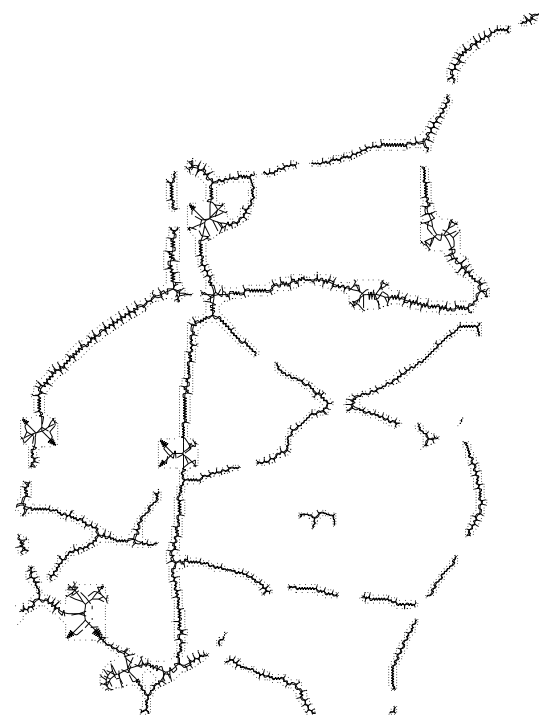

(b) The border (dotted) of the selected object, the dual graph (dashed) and the skeleton (solid)

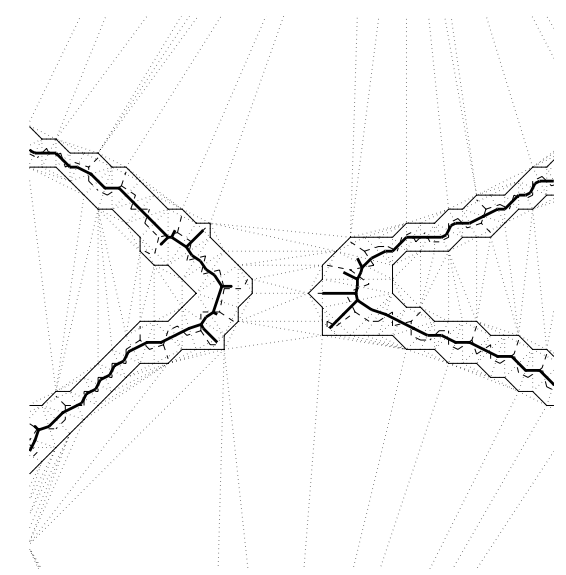

(c) The border (solid), the Delaunay graph (dotted), the dual graph (dashed) and the skeleton (solid thick) of a small portion of the image

Figure 6. The medial axis of a scanned image of the map of the North of Denmark

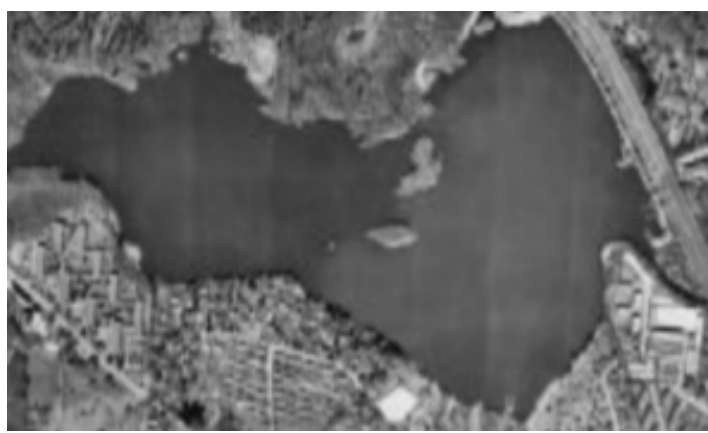

(a) Original image

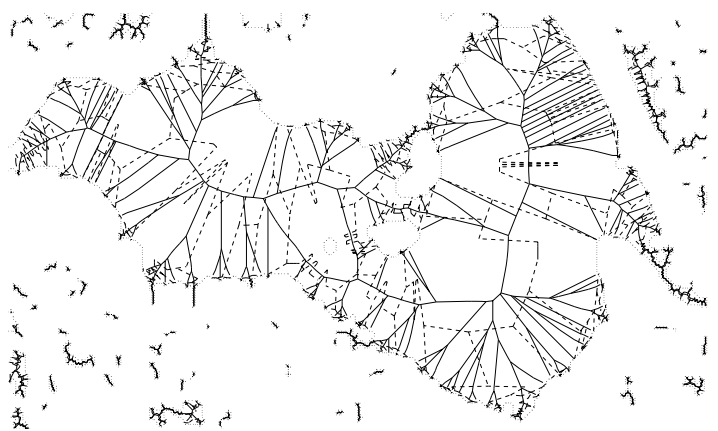

(b) The border (dotted) of the selected object, the dual graph (dashed) and the skeleton (solid)

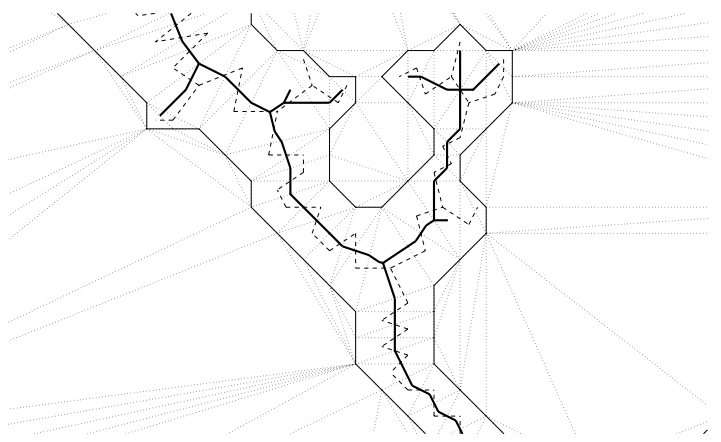

(c) The border (solid), the Delaunay graph (dotted), the dual graph (dashed) and the skeleton (solid thick) of a small portion of the image

Figure 7. The medial axis of a remote sensing image of a lake of Lyngby (Denmark)

[7] R. L. Ogniewicz, "Skeleton-space: A multiscale shape description combining region and boundary information," in Proceedings of Computer Vision and Pattern Recognition, 1994, 1994, pp. 746-751. 
[8] D. Comaniciu and P. Meer, "Robust analysis of feature spaces: color image segmentation," in Proceedings of the 1997 Conference on Computer Vision and Pattern Recognition (CVPR '97). Washington, DC, USA: IEEE Computer Society, 1997, pp. $750-755$.

[9] P. Green and R. Sibson, "Computing dirichlet tessellations in the plane," The Computer Journal, vol. 21, no. 2, pp. 168173, 1977.

[10] D. I. Comaniciu, "Non-parametric robust methods for computer vision," Ph.D. dissertation, Rutgers, The State University of New Jersey, 2000. 